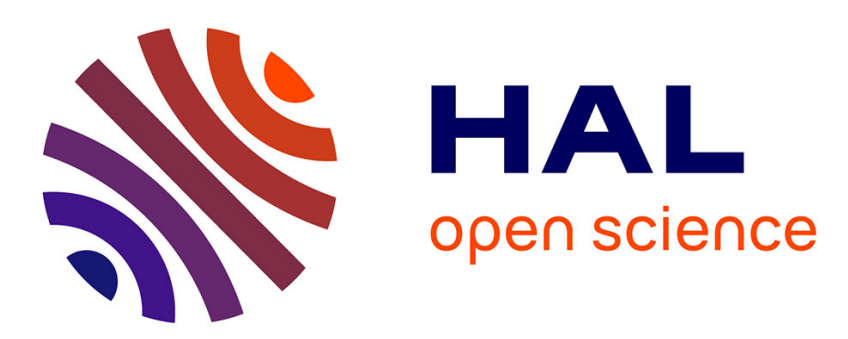

\title{
Studying co-movements in large multivariate data prior to multivariate modelling
}

Gianluca Cubadda, Alain Hecq, Franz C. Palm

\section{To cite this version:}

Gianluca Cubadda, Alain Hecq, Franz C. Palm. Studying co-movements in large multivariate data prior to multivariate modelling. Econometrics, 2009, 148 (1), pp.25. 10.1016/j.jeconom.2008.08.026 . hal-00558630

\section{HAL Id: hal-00558630 \\ https://hal.science/hal-00558630}

Submitted on 23 Jan 2011

HAL is a multi-disciplinary open access archive for the deposit and dissemination of scientific research documents, whether they are published or not. The documents may come from teaching and research institutions in France or abroad, or from public or private research centers.
L'archive ouverte pluridisciplinaire HAL, est destinée au dépôt et à la diffusion de documents scientifiques de niveau recherche, publiés ou non, émanant des établissements d'enseignement et de recherche français ou étrangers, des laboratoires publics ou privés. 


\section{Accepted Manuscript}

Studying co-movements in large multivariate data prior to multivariate

modelling

Gianluca Cubadda, Alain Hecq, Franz C. Palm

PII:

S0304-4076(08)00118-8

DOI:

10.1016/j.jeconom.2008.08.026

Reference:

ECONOM 3074

To appear in: Journal of Econometrics

Received date: 3 September 2007

Revised date: 20 May 2008

Accepted date: 26 August 2008

Please cite this article as: Cubadda, G., Hecq, A., Palm, F.C., Studying co-movements in large multivariate data prior to multivariate modelling. Journal of Econometrics (2008),

doi:10.1016/j.jeconom.2008.08.026

This is a PDF file of an unedited manuscript that has been accepted for publication. As a service to our customers we are providing this early version of the manuscript. The manuscript will undergo copyediting, typesetting, and review of the resulting proof before it is published in its final form. Please note that during the production process errors may be discovered which could affect the content, and all legal disclaimers that apply to the journal pertain. 


\title{
Studying Co-movements in Large Multivariate Data Prior to Multivariate Modelling*
}

\author{
Gianluca Cubadda \\ Università di Roma "Tor Vergata" \\ Alain Hecq \\ Maastricht University \\ Franz C. Palm ${ }^{\dagger}$ \\ Maastricht University
}

September 23, 2008

\begin{abstract}
For non-stationary vector autoregressive models (VAR hereafter, or VAR with moving average, VARMA hereafter), we show that the presence of common cyclical features or cointegration leads to a reduction of the order of the implied univariate autoregressive-moving average (ARIMA hereafter) models. This finding can explain why we identify parsimonious univariate ARIMA models in applied research although VAR models of typical order and dimension used in macroeconometrics imply nonparsimonious univariate ARIMA representations.

Next, we develop a strategy for studying interactions between variables prior to possibly modelling them in a multivariate setting. Indeed, the similarity of the autoregressive roots will be informative about the presence of co-movements in a set of multiple time series. Our results justify both the use of a panel setup with homogeneous autoregression and heterogeneous cross-correlated vector moving average errors and a factor structure, and the use of cross-sectional aggregates of ARIMA series to estimate the homogeneous autoregression.

Keywords: Interactions, multiple time series, co-movements, ARIMA, cointegration, common cycles, dynamic panel data. JEL: C32
\end{abstract}

*Previous drafts of this paper were presented at the Joint Statistical Meetings in Salt Lake City, 62nd European Meeting of the Econometric Society in Budapest, and EC2 Conference on Advanced Time Series Econometrics in Faro. We wish to thank two anonymous referees and the editor Arnold Zellner for useful comments. The usual disclaimers apply.

${ }^{\dagger}$ Corresponding author: Franz C. Palm, Maastricht University, Department of Quantitative Economics, P.O.Box 616, 6200 MD Maastricht, The Netherlands. Email: f.palm@ke.unimaas.nl. 


\section{Introduction}

The analysis of interactions between individuals, groups of agents, organizations, regions or countries is a key objective of scientific inquiry. Huge collections of data (national accounts for virtually all countries, unemployment data for hundreds of types of jobs, prices for thousands of products, assets and services, purchases by individual consumers, environmental data on CO2 emissions and climate change, on health care, education,...) are almost instantaneously available at relatively low cost. The IMF and the World Bank publish statistical information for 150 countries. In the European Union, starting for the six founding countries, Eurostat now collects and analyzes hundreds of variables for the 27 member countries. The availability of sets of data for a large number of individual entities and the importance of understanding individual behavior and interaction between individuals have created a demand for methods to analyze these types of phenomena using large sets of data.

To capture interactions between a large set of time series requires imposing structure on the models used in the analysis. Examples of structure often imposed are the dynamic factor models put forward by Forni et al. (2000) and by Stock and Watson (2002). Another example is the approach of using the common factor structure adopted in the recent literature on dynamic macro-panels to account for cross-sectional correlation (e.g. Bai and Ng, 2004). In these approaches, the common factors usually account for the impact of changes in the environment that is largely exogenous and in which individual entities have to take decisions and to interact. Individual specificities are often accounted for by including individual effects.

Alternatively, partial systems are built for country-specific analyses or for a specific variable such as for instance GDP for a subset of countries or regions. An often adopted framework to analyze a limited number of time series is the vector autoregressive model (VAR hereafter). A VAR, allowing all variables to be endogenous, can only be implemented for small systems, with typically five or six variables. Still, in such cases, the number of parameters is large. To deal with the dimensionality problem in VARs, different approaches have been adopted such as inter alia Bayesian analysis, simulation-based techniques, separability assumptions (as for instance used in panel data models when they are accompanied by the homogeneity assumption of the autoregressive parameters across units), automatic selection by deleting non-significant coefficients and reduced-rank regressions.

In this paper we take a different route and make two new contributions to the literature. Instead of designing a system which captures interactions between entities, we first address the question what the implications are of the presence of common factors in a possibly large VAR system on the marginal processes for individual entities. The common factor structure could arise from the presence of common cyclical or

other features (see e.g. Engle and Kozicki, 1993). For non-stationary series, a common factor structure could result from the presence of cointegration, that is from the occurrence of common stochastic trends. The first main contribution of the paper is that the presence of common factors or common features in a VAR leads to a reduction of the order of the implied univariate autoregressive-moving average (ARIMA hereafter) schemes for the individual series. In a way, individual series keep a print of the system as a whole. Second, we propose a strategy, that allows to identify the print of common features from individual data, that is to study aspects of interactions between individual variables without or prior to modelling these interactions 
in a multivariate framework.

Before implementing this strategy, we derive the implications of the presence of a common factor structure for the univariate processes implied by a VAR, both in absence of and under exogeneity restrictions. More precisely, in Section 2, we extend results by Zellner and Palm (1974) by showing that VARs with reduced rank restrictions coming from short- or long-run co-movements (see inter alia Vahid and Engle, 1993, and Ahn, 1997) offer an alternative explanation to the well known paradox in time series: on the one hand, small multivariate systems imply non-parsimonious individual ARIMA processes whereas on the other hand empirically selected and estimated univariate models are generally of low order.

Section 3 proposes and evaluates an estimation strategy based upon the common univariate autoregressive parameters of series generated by the same VAR system. Indeed, a homogeneous panel framework with common autoregressive parameters is the by-product of a large VAR. We propose to use cross-sectional aggregates to estimate these common autoregressive parameters, and we evaluate this estimation strategy in a small Monte Carlo experiment. Section 4 focuses on the link between the growth rates of GDP among Latin American economies. The occurrence of common autoregressive parts for the individual series is used as an indication that these series exhibit the same common features and therefore are subject to the same co-movements. This indication is then formally tested and found to be supported by the information in the data. A final section concludes and provides suggestions for further research.

\section{Multivariate and implied univariate time series models}

The aim of this section is to present new results on the relations between univariate and multivariate time series models. In particular, Subsection 2.1 briefly reviews the paradox, namely that VARs of typical order and dimension used in macroeconometrics imply non-parsimonious univariate ARMA representations, which are rarely observed in empirical applications. Subsection 2.2 shows how VARs with certain reduced rank structures can solve this paradox, and Subsection 2.3 specializes the results to the case of block-diagonal and block-triangular VARs. Moreover, Subsection 2.4 explores the implications of short-run co-movements for the vector moving average (VMA hereafter) component of the so-called final equation representation of a VAR model (Zellner and Palm, 1974). The findings may explain why MA orders of univariate models are often found to be smaller than those theoretically implied by multivariate models. Finally, Subsection 2.5 illustrates concepts and methods with an empirical analysis of the relationship between quarterly growth rates of the industrial production indexes in the US and Canada.

\subsection{The paradox}

The literature on the link between multivariate time series and the behavior of univariate variables stresses the fact that univariate ARIMA analyses provide tools for the diagnostic testing of VAR (or VARMA) models. Important contributions in this area are the monograph by Quenouille (1957) and papers by Zellner and Palm (1974, 1975, 2004), Palm and Zellner (1980), Palm (1977) or Maravall and Mathis (1994). Some textbooks devote a few pages to that issue (e.g. Franses (1998, 198-199) or Lütkepohl (2005, 494-495)). 
More formally, let us first consider the stationary ${ }^{1}$ multivariate VARMA process for an $n$ vector of time series $z_{t}=\left(z_{1 t}, \ldots, z_{n t}\right)^{\prime}$, and without deterministic terms for simplicity:

$$
\Phi(L) z_{t}=\Theta(L) \varepsilon_{t}, \quad t=1, \ldots, T
$$

where $\Phi(L)$ and $\Theta(L)$ are finite order polynomial coefficient matrices with the usual lag operator $L$ such that $L z_{t}=z_{t-1}$. The sequence of $\varepsilon_{t}$ is a multivariate white noise process with each $\varepsilon_{t} \sim N(0, \Sigma)$. The VARMA model in (1) encompasses several useful specifications. For example, if $\Phi(L) \equiv I$ we have the vector moving average representation or $\operatorname{VMA}$; if $\Theta(L) \equiv I$ we have a $\operatorname{VAR}(p)$. Let us concentrate the analysis on $\operatorname{VAR}$ models of order $p$, denoted $\operatorname{VAR}(p)$, one of the cornerstone specification in empirical macroeconometrics:

$$
\Phi(L) z_{t}=\varepsilon_{t} .
$$

Following Zellner and Palm (1974), the univariate representation of elements of $z_{t}$ can be obtained by premultiplying both sides of $(2)$ by $\Phi(L)^{a d j}$, the adjoint matrix (or the adjugate) associated with $\Phi(L)$, in order to obtain the "final equations" (FEs henceforth):

$$
\operatorname{det}[\Phi(L)] z_{t}=\Phi(L)^{a d j} \varepsilon_{t}
$$

where the determinant $\operatorname{det}[\Phi(L)]$ is a scalar finite order polynomial in $L$. This means that each series is a finite order $\operatorname{ARMA}\left(p^{*}, q^{*}\right)$, with the same lag structure and the same coefficients for the autoregressive part for every series, although the multivariate system is a finite order $\operatorname{VAR}(p)$. For instance for the $i$ th element of $z_{t}$ we have

$$
\operatorname{det}[\Phi(L)] z_{i t}=\Phi_{i .}(L)^{a d j} \varepsilon_{t}=\theta_{i}(L) u_{i t},
$$

$\Phi_{i .}(L)^{a d j}$ denoting the $i$ th row of the matrix $\Phi(L)^{a d j}, \theta_{i}(L)$ is a scalar polynomial in $L$ and $u_{i t}$ is a scalar innovation with respect to the past of $z_{i t}$. This recognition and the compatibility of these $p^{*}$ and $q^{*}$ with $n$ and $p$ of the $\operatorname{VAR}(p)$ is the first step of an approach that has been developed in Zellner and Palm $(1974,1975)$ as a general modelling strategy called SEMTSA (Structural Economic Modelling Time Series Analysis). In the first stage of this approach, information from univariate schemes is used to restrict the dynamics of the structural model. However, given the conditions associated with that first diagnostic checking, one often faces a paradoxical situation: a small order VAR with few series already generates univariate ARMA with large $p^{*}$ and $q^{*}$, an implication that is rejected when tested on economic data where one usually finds quite parsimonious models with low order autoregressive and moving average polynomials.

Indeed, an $n$ dimensional $\operatorname{VAR}(p)$ would imply at most individual $\operatorname{ARMA}(n p,(n-1) p)$ processes. This well known result is simply due to the fact that $\operatorname{det}[\Phi(L)]$ contains by construction up to $L^{n p}$ terms and the adjoint matrix is a collection $(n-1) \times(n-1)$ cofactor matrices, each of the matrix elements can contain the terms $1, L, . . L^{p}$. But whatever the simplicity of that result, it leads to implausible univariate models for

\footnotetext{
${ }^{1}$ The generalisation to non-stationarity processes will be considered later in this section.
} 
most economic series. ${ }^{2}$ This is illustrated in the following numerical example.

Example 1 Let us consider the bivariate VAR(2), $\Phi(L) z_{t}=\left(I-\Phi_{1} L-\Phi_{2} L^{2}\right) z_{t}=\varepsilon_{t}$ with the following numerical values

$$
\Phi(L)=\left[\begin{array}{ll}
1 & 0 \\
0 & 1
\end{array}\right]-\left[\begin{array}{cc}
-0.1 & -0.21 \\
0.7 & 0.76
\end{array}\right] L-\left[\begin{array}{cc}
0.3 & 0.8 \\
0.3 & -0.8
\end{array}\right] L^{2}
$$

the determinant of which is $-0.48 L^{4}-0.189 L^{3}+0.571 L^{2}-0.66 L+1$ and the adjoint matrix such that

$$
\Phi(L)^{a d j}=\left[\begin{array}{cc}
1-0.76 L+0.8 L^{2} & -0.21 L+0.8 L^{2} \\
0.7 L+0.3 L^{2} & 1+0.1 L-0.3 L^{2}
\end{array}\right]
$$

The two implied individual series are $\operatorname{ARMA(4,2).}$

Wallis (1977) has proposed two explanations to solving this paradox. The first solution is to unravel and get rid of common roots in $\operatorname{det}[\Phi(L)]$ and $\Phi(L)^{a d j}$. This requirement of minimal representation is not always trivial to implement in practice. The cancelling of common factors occurs for instance when the autoregressive matrix $\Phi(L)$ is diagonal, block-diagonal, triangular or block-triangular as pointed out by Zellner and Palm (1974) and Palm (1977). Under a block-triangular structure and a corresponding block-diagonality of $\Sigma$, a subset of $z_{t}$ is exogenous. The second intuition raised by Wallis is based on the observation that coefficients close to but different from zero in the implied ARMA model might give the feeling that models are more parsimonious than they theoretically should be. This explanation is always inherent in most of the papers by Zellner and Palm where economic theories are tested and restrictions are imposed on the system which are compatible with implied individual ARMA models. Also the strategies using algorithms to systematically delete non-significant coefficients in VAR models (see inter alia Brüggemann et al. (2003)) fall into this setting. The next subsection provides an alternative explanation for solving that paradox thanks to the use of short-run co-movements and reduced rank models.

\subsection{Solving the paradox under common features}

We introduce a new framework aimed at understanding the gap between implied individual ARMA processes for VARs and the estimated univariate models that we get in empirical studies. More specifically, we investigate the implications of the presence of common features such as common cyclical features and common trends in a multivariate dynamic model for the order of the marginal processes of the individual series.

The strongest form of common cyclical features is the notion of serial correlation common feature (henceforth, SCCF) proposed by Engle and Kozicki (1993) and Vahid and Engle (1993). ${ }^{3}$ In this framework, series $z_{t}$ have $s \mathrm{SCCF}$ relationships if there exists an $n \times s(s<n)$ matrix $\delta$ with full column rank and such that $\delta^{\prime} \Phi(L) z_{t}=\delta^{\prime} z_{t}=\delta^{\prime} \varepsilon_{t}$ in (2). Hence, SCCF implies that impulse response functions of series in $z_{t}$ are collinear.

\footnotetext{
${ }^{2}$ This result generalizes to individual $\operatorname{ARMA}(n p,(n-1) p+q)$ for $\operatorname{VARMA}(p, q)$ processes (see e.g. Lütkepohl (2005)).

3 This model is also known as a scalar component model of order zero or SCM $(0,0)$ using the terminology of Tiao and Tsay (1989) or white noise direction codependence by Gouriéroux and Peaucelle (1989).
} 
Although convenient in terms of parsimony and economic interpretation of shocks' transmission mechanisms (see, inter alia, Centoni et al., 2007), assumptions underlying SCCF may be too strong. Indeed, the definition of SCCF does not take account of the possibility that common serial correlation is present among non-contemporaneous elements of series $z_{t}$ (see e.g. Ericsson, 1993). In order to overcome this limitation, several variants of the SCCF were proposed, see, inter alia, Vahid and Engle (1997), Schleicher (2007), Hecq et al. (2006), Cubadda (2007), and Cubadda and Hecq (2001). In particular, these latter authors assume that there exists an $n \times s$ polynomial matrix such that $\delta^{\prime}(L) z_{t} \equiv\left(\delta_{0}+\delta_{1} L\right)^{\prime} z_{t}=\delta_{0}^{\prime} \varepsilon_{t}$. This model is a polynomial SCCF model of order one, which is denoted PSCCF(1). ${ }^{4}$ The notion of PSCCF can be generalized to account for adjustment delays of $m$ periods instead of one lag as in the PSCCF(1). In this case the polynomial matrix $\delta(L)$ is of order $m$, where $m \leq p$, a model denoted $\operatorname{PSCCF}(m)$ (see Cubadda and Hecq (2001) for details).

For simplicity reasons, we start with the most parsimonious model, in which there exists a SCCF matrix. We first illustrate the consequences of the presence of one SCCF relationship in a bivariate example with $p=2$ before generalizing to the $n$ dimensional case for any polynomial order $p$ and other forms of non contemporaneous co-movements (e.g. PSCCF).

Example 2 Let us consider the polynomial matrix $\Phi(L)$ of the Example 1 but now there exists a SCCF relationship with a cofeature vector $\delta^{\prime}=(1: 1)$ for

$$
\Phi(L)=\left[\begin{array}{ll}
1 & 0 \\
0 & 1
\end{array}\right]-\left[\begin{array}{cc}
-0.1 & -0.2 \\
0.1 & 0.2
\end{array}\right] L-\left[\begin{array}{cc}
0.2 & 0.6 \\
-0.2 & -0.6
\end{array}\right] L^{2}
$$

Because the second and the third coefficient matrices $\Phi_{1}$ and $\Phi_{2}$ are of reduced rank with the same left null space, it follows that $\delta^{\prime} \Phi(L)=\delta^{\prime}$. The determinant of $\Phi(L)$ in (4) is $0.4 L^{2}-0.1 L+1$ and the adjoint matrix is

$$
\Phi(L)^{a d j}=\left[\begin{array}{cc}
-0.2 L+0.6 L^{2}+1 & -0.2 L+0.6 L^{2} \\
0.1 L-0.2 L^{2} & 0.1 L-0.2 L^{2}+1
\end{array}\right] .
$$

This implies that the two series are at most $A R M A(2,2)$ and not $A R M A(4,2)$ as in Example 1.

More generally, Table 1 summarizes the reduction of the individual ARMA orders due to common feature restrictions. Basically, Table 1 documents that multivariate systems with additional commonality deliver more parsimonious, and therefore empirically more plausible, ARMA models than those implied by unrestricted VAR models. For instance, in a small VAR system with four variables and two lags, implied models are at most $\operatorname{ARMA}(8,6)$ while they would reduce to $\operatorname{ARMA}(2,2)$ models in the presence of a unique common cycle, that is, with $s=3$. This application to Quenouille's results, who already stressed the impact of rank deficiency, is one of the diagnostic tools for data-admissibility that we want to formalize and emphasize. Note also that additional zero coefficient restrictions on the common factors and/or the loadings may reduce

\footnotetext{
${ }^{4}$ Notice that a SCCF relationship involving, for instance, $z_{1 t}$ and $z_{2 t-1}$, where $z_{t}=\left(z_{1 t}^{\prime}, z_{2 t}^{\prime}\right)^{\prime}$, is a particular case of a $\operatorname{PSCCF}(1)$.
} 
these implied maximal orders. This means that the $\operatorname{ARMA}(2,2)$ obtained under short-run co-movements can be more parsimonious with additional exclusion restrictions.

Let us formally prove the results that are reported in Table 1.

TABLE 1

Maximum ARMA orders of univariate series generated by an $n$-dimensional $\mathrm{I}(0) \operatorname{VAR}(p)$ with $s$ cofeature restrictions

\begin{tabular}{lcc}
\hline Models & AR order & MA order \\
\hline \hline $\operatorname{VAR}(p)$ & $n p$ & $(n-1) p$ \\
$\operatorname{SCCF}$ & $(n-s) p$ & $(n-s) p$ \\
$\operatorname{PSCCF}(1)$ & $(n-s) p+s$ & $(n-s) p+(s-1)$ \\
$\operatorname{PSCCF}(m)$ & $(n-s) p+s m$ & $(n-s) p+(s-1) m$ \\
\hline
\end{tabular}

Proposition 3 Stationary VAR with $s$ SCCF. In an n-dimensional stationary VAR $(p)$ with $s$ SCCF, the individual ARMA processes have: (i) AR orders not larger than $(n-s) p$; (ii) MA orders not larger than $(n-s) p$.

Proof. Let us rewrite equation (2) as follows

$$
Q(L) x_{t}=e_{t}
$$

where $x_{t}=M z_{t}, e_{t}=M \varepsilon_{t}, Q(L)=M \Phi(L) M^{-1}, M^{\prime} \equiv\left[\delta: \delta_{\perp}\right]$, and $\delta_{\perp}$ is the orthogonal complement. Given that $x_{t}$ is a non-singular linear transformation of $z_{t}$, the maximum $A R$ and MA orders of the univariate representation of elements of $z_{t}$ must be the same as those of elements of $x_{t}$. Since $M^{-1}=\left[\bar{\delta}: \bar{\delta}_{\perp}\right]$, where $\bar{\delta}=\delta\left(\delta^{\prime} \delta\right)^{-1}$, and $\bar{\delta}_{\perp}=\delta_{\perp}\left(\delta_{\perp}^{\prime} \delta_{\perp}\right)^{-1}$, we have

$$
Q(L)=\left[\begin{array}{cc}
I_{s} & 0_{s \times(n-s)} \\
\delta_{\perp}^{\prime} \Phi(L) \bar{\delta} & \delta_{\perp}^{\prime} \Phi(L) \bar{\delta}_{\perp}
\end{array}\right]
$$

from which it easily follows that $\operatorname{det}[Q(L)]=\operatorname{det}\left[\delta_{\perp}^{\prime} \Phi(L) \bar{\delta}_{\perp}\right]$ is a polynomial of order $(n-s) p$. Hence, the univariate $A R$ order of each element of $z_{t}$ is, at most, $(n-s) p$. To prove (ii), let $P(L)$ denote a submatrix of $Q(L)$ that is formed by deleting one of the first $s$ rows and one of the first $s$ columns of $Q(L)$. We can partition $P(L)$ as follows

$$
P(L)=\left[\begin{array}{cc}
P_{11} & P_{12} \\
P_{21}(L) & P_{22}(L)
\end{array}\right] .
$$

Now, $P_{11}$ is a $(s-1) \times(s-1)$ identity matrix, $P_{12}$ is a $(s-1) \times(n-s)$ matrix of zeros, $P_{21}(L)$ is a $(n-s) \times(s-1)$ polynomial matrix of order $p$, and $P_{22}(L)$ is a $(n-s) \times(n-s)$ polynomial matrix of order $p$. Hence, $\operatorname{det}[P(L)]=\operatorname{det}\left[P_{11}\right] \operatorname{det}\left[P_{22}(L)\right]$, which tells us that $\operatorname{det}[P(L)]$ is of order $(n-s) p$. Since cofactors associated with the blocks of $Q(L)$ different from $P_{11}$ are polynomials of degree not larger than $(n-s) p$, we conclude that the univariate MA order of each element of $z_{t}$ is, at most, $(n-s) p$. 
Proposition 4 Stationary VAR with s PSCCF(1). In an n stationary $\operatorname{VAR}(p)$ with s PSCCF(1) the individual ARMA processes have: (i) AR orders not larger than $(n-s) p+s ;($ ii) $M A$ orders not larger than $(n-s) p+(s-1)$.

Proof. Along the same lines of the previous proof, let us consider $R(L)=M_{0} \Phi(L) M_{0}^{-1}, M_{0}^{\prime} \equiv\left[\delta_{0}: \delta_{0 \perp}\right]$, and $M_{0}^{-1} \equiv\left[\bar{\delta}_{0}: \bar{\delta}_{0 \perp}\right]$. Then, we have

$$
R(L)=\left[\begin{array}{cc}
\delta^{\prime}(L) \bar{\delta}_{0} & \delta_{1}^{\prime} \bar{\delta}_{0 \perp} L \\
\delta_{0 \perp}^{\prime} \Phi(L) \bar{\delta}_{0} & \delta_{0 \perp}^{\prime} \Phi(L) \bar{\delta}_{0 \perp}
\end{array}\right]
$$

Hence,

$$
\operatorname{det}[R(L)]=\operatorname{det}\left[\delta^{\prime}(L) \bar{\delta}_{0}\right] \operatorname{det}\left[\delta_{0 \perp}^{\prime} \Phi(L) \bar{\delta}_{0 \perp}-\delta_{0 \perp}^{\prime} \Phi(L) \bar{\delta}_{0}\left(\delta^{\prime}(L) \bar{\delta}_{0}\right)^{-1} \delta_{1}^{\prime} \bar{\delta}_{0 \perp} L\right] .
$$

Writing $\left(\delta^{\prime}(L) \bar{\delta}_{0}\right)^{-1}=\left(\delta^{\prime}(L) \bar{\delta}_{0}\right)^{\text {adj }} / \operatorname{det}\left[\delta^{\prime}(L) \bar{\delta}_{0}\right]$ and substituting it above we get

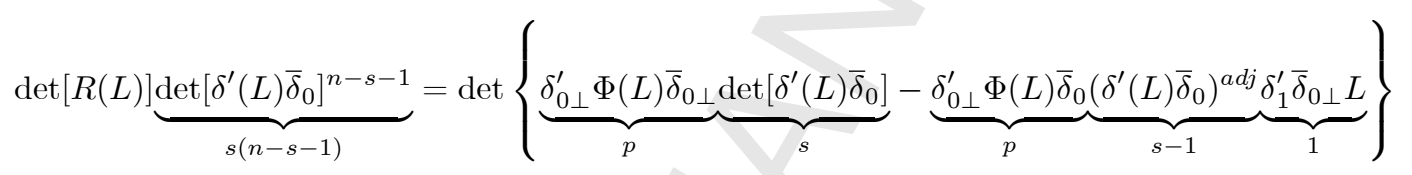

from which follows that the degree of the polynomial of $\operatorname{det}[R(L)]$ is, at most, equal to $(n-s) p+s$. Regarding the order of the MA component, let us denote $G(L)$ a submatrix of $R(L)$ that is formed by deleting one of the first $s$ rows and one of the first s columns of $R(L)$. We can partition $G(L)$ as follows

$$
G(L)=\left[\begin{array}{cc}
G_{11}(L) & G_{12} L \\
G_{21}(L) & G_{22}(L)
\end{array}\right]
$$

where $G_{11}(L)$ is a $(s-1) \times(s-1)$ polynomial matrix of order $1, G_{12}$ is a $(s-1) \times(n-s)$ matrix, $G_{21}(L)$ is a $(n-s) \times(s-1)$ polynomial matrix of order $p$, and $G_{22}(L)$ is a $(n-s) \times(n-s)$ polynomial matrix of order p. Hence, following a similar reasoning as above, we conclude that the individual MA order is, at most, $(n-s) p+(s-1)$.

These results can be easily generalized for the $\operatorname{PSCCF}(m)$ case as reported in Table 1. Also note that we do not consider a mixed model that can have both SCCF and PSCCF relationships but results can be trivially deduced from results beneath.

The above propositions can be extended to the case of an I(1) VAR. Let us consider the vector error correction model (VECM henceforth) of variables $z_{t}$

$$
\Gamma(L) \Delta z_{t}=\alpha \beta^{\prime} z_{t-1}+\varepsilon_{t},
$$

where $\Delta=(1-L), \Gamma(L)=I_{n}-\sum_{i=1}^{p-1} \Gamma_{i} L^{i}, \Gamma_{i}=-\sum_{j=i+1}^{p} \Phi_{j}$ for $i=1,2, \ldots, p-1, \alpha$ and $\beta$ are fullcolumn rank $n \times r(r<n)$ matrices such that $\Phi(1)=-\alpha \beta^{\prime}$ and $\alpha_{\perp}^{\prime} \Gamma(1) \beta_{\perp}$ has full rank. The process $z_{t}$ is cointegrated of order $(1,1)$, denoted by $\mathrm{CI}(1,1)$, the columns of $\beta$ span the cointegrating space, the elements of $\alpha$ are the corresponding adjustment coefficients, see e.g. Johansen (1996). 
A well known implication of cointegration is that $(n-r-1)$ unit roots are common to the individual AR and the MA polynomial. Let us formalize this result in order to later extend it to different forms of common cyclical features. To do so, consider again equation (2) and apply the transformation

$$
B(L) y_{t}=u_{t}
$$

where $y_{t}=P z_{t}, u_{t}=P \varepsilon_{t}, B(L)=P \Phi(L) P^{-1}$, and $P^{\prime} \equiv\left[\beta: \beta_{\perp}\right]$. Again, the maximum AR and MA orders of the univariate representation of elements of $z_{t}$ are the same as those of elements of $y_{t}$. Since $\beta^{\prime} z_{t}$ is $\mathrm{I}(0)$ and $\beta_{\perp}^{\prime} z_{t}$ is $\mathrm{I}(1)$, we can partition $B(L)$ as follows

$$
B(L)=\left[\begin{array}{cc}
B_{11}(L) & \Delta B_{12}(L) \\
B_{21}(L) & \Delta B_{22}(L)
\end{array}\right] .
$$

We have that

$$
\operatorname{det}[B(L)]=\Delta^{n-r} \operatorname{det}\left[B_{22}(L)\right] \operatorname{det}\left[B_{11}(L)-B_{12}(L) B_{22}(L)^{-1} B_{21}(L)\right] \equiv \Delta^{n-r} \operatorname{det}\left[B_{22}(L)\right] \operatorname{det}\left[B^{*}(L)\right]
$$

Since $B_{22}(1) \neq 0$, and $B^{*}(1)=B_{11}(1) \neq 0$, there are $(n-r)$ unit roots in $\operatorname{det}[B(L)]$. However, since $\beta_{\perp}^{\prime} z_{t}$ is $\mathrm{I}(1)$, there must be $(n-r-1)$ unit roots in the univariate MA polynomials of elements of $\beta_{\perp} z_{t}$. Hence, the AR and MA orders of elements of $z_{t}$ respectively are, at most, $n(p-1)+r+1$ and $(n-1)(p-1)+r$.

For the VECM in (6), the existence of $s$ SCCF's implies that there exists an $n \times s$ full-rank matrix $\delta$ such that $\delta^{\prime} \Delta z_{t}=\delta^{\prime} \varepsilon_{t}$ or equivalently

$$
\delta^{\prime} \Phi(L)=\delta^{\prime} \Delta
$$

As shown by Vahid and Engle (1993), the presence of $s$ SCCF's is equivalent to the existence of $(n-s)$ common and synchronous cycles ${ }^{5}$.

The existence of $s$ PSCCF(1) relationships requires, instead, that there exists an $n \times s$ full-rank matrix $\delta_{0}$ such that $\delta_{0}^{\prime} \Delta z_{t}+\delta_{1}^{\prime} \Delta z_{t-1}=\delta_{0}^{\prime} \varepsilon_{t}$ where $\delta_{1}=-\Gamma_{1}^{\prime} \delta_{0}$ or equivalently

$$
\delta(L)^{\prime} \Phi(L)=\delta_{0}^{\prime} \Delta
$$

The presence of the PSCCF(1) has an interesting implication for the cycles of series $z_{t}$. Indeed, Cubadda and Hecq (2001) proved that the same PSCCF(1) relationships cancel the dependence from the past of both the first differences and cycles of series $z_{t}$.

Finally, the existence of $s$ weak form SCCF (WF henceforth) implies that there exists an $n \times s$ full-rank matrix $\tilde{\delta}$ such that $\tilde{\delta}^{\prime} \Delta z_{t}-\tilde{\delta}^{\prime} \alpha \beta^{\prime} z_{t-1}=\tilde{\delta}^{\prime} \varepsilon_{t}$ (Hecq et al., 2006) or equivalently

$$
\tilde{\delta}(L)^{\prime} \Phi(L)=\tilde{\delta}^{\prime}
$$

\footnotetext{
${ }^{5}$ In this framework, cycles are the remainder of a vector $\mathrm{I}(1)$ process after subtracting the (possibly common) random-walk components.
} 
where $\tilde{\delta}(L)=\tilde{\delta}+\tilde{\delta}_{1} L, \tilde{\delta}_{1}=-\left(\beta \alpha^{\prime}+I_{n}\right) \tilde{\delta}$. Interestingly, the same WF relationships cancel the dependence from the past of both the levels and cycles of series $z_{t}$ (Cubadda, 2007). Hence, we can interpret the WF as an analogous property to the $\operatorname{PSCCF}(1)$ that applies to the levels rather than to the differences of series $z_{t}$.

Table 2 reports the largest ARIMA orders of individual series that are generated by a VECM with common feature restrictions. Let us formally prove these results.

TABLE 2

Maximum ARMA orders of univariate series generated by an $n$-dimensional $\mathrm{CI}(1,1) \operatorname{VAR}(p)$ with $s$ cofeature restrictions

\begin{tabular}{lccc}
\hline Models & AR order & Integration order & MA order \\
\hline \hline $\operatorname{VAR}(p)$ & $n(p-1)+r$ & 1 & $(n-1)(p-1)+r$ \\
$\operatorname{SCCF}$ & $(n-s)(p-1)+r$ & 1 & $(n-s)(p-1)+r$ \\
$\operatorname{PSCCF}(1)$ & $(n-s)(p-1)+r+s$ & 1 & $(n-s)(p-1)+r+s-1$ \\
$\mathrm{WF}$ & $(n-s)(p-1)+r$ & 1 & $(n-s)(p-1)+r$ \\
\hline
\end{tabular}

Proposition 5 Cointegrated VAR with $\boldsymbol{s}$ SCCF. In a CI(1,1) VAR(p) with s SCCF the individual ARMA processes of the first differences have: (i) AR orders not larger than $(n-s)(p-1)+r$; (ii) MA orders not larger than $(n-s)(p-1)+r$.

Proof. By construction of $M$, and keeping in mind that $\delta^{\prime} \Phi(L)=\delta^{\prime} \Delta$, we notice that

$$
\operatorname{det}[Q(L)]=\operatorname{det}\left[\begin{array}{cc}
\Delta I_{s} & 0_{s \times(n-s)} \\
\delta_{\perp}^{\prime} \Phi(L) \bar{\delta} & \delta_{\perp}^{\prime} \Phi(L) \bar{\delta}_{\perp}
\end{array}\right]=\Delta^{s} \operatorname{det}\left[\delta_{\perp}^{\prime} \Phi(L) \bar{\delta}_{\perp}\right]
$$

is a polynomial of order $(n-s) p+s$. Since $\operatorname{det}[Q(L)]$ is a polynomial of the same order as $\operatorname{det}[\Phi(L)]$, we conclude that the univariate AR order of each element of $z_{t}$ is, at most, $(n-s) p+s$. Note that this result means that the AR part is of order $(n-s)(p-1)+n$. The polynomial degree of the moving average component is obtained along the same line as in Proposition 3 but with $P_{11} \Delta$ instead of $P_{11}$ in (5). Hence, $\operatorname{det}[P(L)]=\operatorname{det}\left[P_{11} \Delta\right] \operatorname{det}\left[P_{22}(L)\right]$, which tells us that $\operatorname{det}[P(L)]$ is of order $(n-s) p+(s-1)$. Since the presence of cointegration implies that $(n-r-1)$ common unit roots cancel out from the individual $A R$ and the MA polynomials, the result follows.

Proposition 6 Cointegrated VAR with s PSCCF(1). In a CI(1,1) VAR(p) with s PSCCF(1) the individual ARMA processes of the first differences have: (i) AR orders not larger than $(n-s)(p-1)+s+r$; (ii) $M A$ orders not larger than $(n-s)(p-1)+r+s-1$.

Proof. Since it is similar to that of Proposition 4, it is not reported to save space.

Proposition 7 Cointegrated VAR with $s$ WF. In a CI(1,1) VAR(p) with $s$ WF the ARMA processes of the first differences have: (i) AR orders not larger than $(n-s)(p-1)+r$; (ii) MA orders not larger than $(n-s)(p-1)+r$. 
Proof. Let us consider $H(L)=\widetilde{M} \Phi(L) \widetilde{M}^{-1}, \widetilde{M}^{\prime} \equiv\left[\widetilde{\delta}: \widetilde{\delta}_{\perp}\right]$, and

$$
H(L)=\left[\begin{array}{cc}
\widetilde{\delta}^{\prime}(L) \widetilde{\delta}\left(\widetilde{\delta}^{\prime} \widetilde{\delta}\right)^{-1} & \widetilde{\delta}_{1}^{\prime} \widetilde{\delta}_{\perp}\left(\widetilde{\delta}_{\perp}^{\prime} \widetilde{\delta}_{\perp}\right)^{-1} L \\
\widetilde{\delta}_{\perp}^{\prime} \Phi(L) \widetilde{\delta}\left(\widetilde{\delta}^{\widetilde{\delta}}\right)^{-1} & \widetilde{\delta}_{\perp}^{\prime} \Phi(L) \widetilde{\delta}_{\perp}\left(\widetilde{\delta}_{\perp}^{\prime} \widetilde{\delta}_{\perp}\right)^{-1}
\end{array}\right]
$$

Hence, the proof is entirely analogous to that of Proposition 4 with the $H(L)$ matrix in place of $R(L)$.

Notice that the results for the VAR in the presence of common features can be extended in a fairly trivial way for the VARMA model. In the presence of common features in a $\operatorname{VARMA}(p, q)$ model, the orders reported in Tables 1 and 2 hold true as well for the AR part whereas the order of the MA parts has to be augmented by $q$ (at most). If common feature restrictions affect the VAR part only, the MA part of the univariate processes is expected to be increased by $q$. The proofs are similar to those reported for the $\operatorname{VAR}(p)$ model except for the presence of the $q$ extra lags in the MA parts. If the common features restrictions affect the MA part of the VARMA model, the increase of the MA part of the implied univariate models might be smaller than $q$.

\subsection{Block-diagonal and block-triangular VARs and the separation hypothesis}

From the results of the previous subsection, there naturally arises the question about block-diagonal and block-triangular systems, namely about a situation in which we can disentangle groups of variables having many within co-movements but being roughly independent of other sets of variables. Indeed, intuition suggests that additional restrictions due to block-diagonality or block-triangularity should induce further simplifications of the individual ARMA structures. A block-triangular structure is required for the absence of Granger-causation between subvectors of $z_{t}$ accordingly partitioned and for strong exogeneity.

For the sake of simplicity, we consider two groups of variables and SCCF restrictions only. The first model under analysis is a block-diagonal stationary $\operatorname{VAR}(p)$ with SCCF within each block of variables $n_{1}$ and $n_{2}$ respectively, with $n_{1}+n_{2}=n$ such that

$$
\Phi(L)=\left[\begin{array}{cc}
\Phi_{11}(L) & 0_{n_{1} \times n_{2}} \\
0_{n_{2} \times n_{1}} & \Phi_{22}(L)
\end{array}\right],
$$

where there exist two full column rank matrices with ranks $s_{1}$ and $s_{2}$ respectively, with $s_{1}+s_{2}=s$ such that $\delta_{1}^{\prime} \Phi_{11}(L)=\delta_{1}^{\prime}$ and $\delta_{2}^{\prime} \Phi_{22}(L)=\delta_{2}^{\prime}$. This is a system with separability in common features (Hecq et al., 2002) with a block-diagonal co-feature matrix such that

$$
\delta=\left[\begin{array}{cc}
\delta_{1} & 0_{n_{1} \times s 2} \\
0_{n_{2} \times s_{1}} & \delta_{2}
\end{array}\right] .
$$

In view of Table 1, the maximum orders for the implied univariate ARMA processes are $\left(n_{1}+n_{2}-s_{1}-s_{2}\right) p$ for both the AR and MA components, assuming the same polynomial order $p$ for each group. With different polynomial orders $p_{1}$ and $p_{2}$, these orders become $\left(n_{1}-s_{1}\right) p_{1}+\left(n_{2}-s_{2}\right) p_{2}$ respectively. However, it is clear that these orders can be further reduced due to the presence of blocks of zeros. Because this leads to 
the presence of common roots between the AR and MA parts, the maximum orders of both AR and MA components are $\left(n_{1}-s_{1}\right) p_{1}$ for the first block and $\left(n_{2}-s_{2}\right) p_{2}$ for the second group of series.

Due to separation in the form of block-diagonality of $\Phi(L)$, the implied univariate ARMA-processes need not to have identical AR polynomials. In fact, the model allows for inter-block AR parameter heterogeneity and intra-block AR parameter homogeneity. Note that this form of separation is often used when studying data for a set of countries using a panel-data framework. In some panel-data studies, a single variable is analyzed for a set of countries which can be clustered in groups with intergroup block-diagonality of $\Phi(L)$ combined with intragroup homogeneity so that the implied univariate ARMA models for a given group have identical AR polynomials.

The second model is a block-triangular VAR such that

$$
\Phi(L)=\left[\begin{array}{cc}
\Phi_{11}(L) & \Phi_{12}(L) \\
0_{n_{2} \times n_{1}} & \Phi_{22}(L)
\end{array}\right]
$$

with $\delta_{1}^{\prime}\left[\Phi_{11}(L): \Phi_{12}(L)\right]=\delta_{1}^{\prime}$ and $\delta_{2}^{\prime} \Phi_{22}(L)=\delta_{2}^{\prime}$. The situation is similar as before because $\delta_{\perp}$ is still block-diagonal. However fewer cancellations of common roots are observed and the implied models are $\left(n_{1}-s_{1}\right) p_{1}+\left(n_{2}-s_{2}\right) p_{2}$ for block 1 and $\left(n_{2}-s_{2}\right) p_{2}$ in the block 2 .

In this case, the AR polynomials of the two blocks could have factors in common which are identical to the AR polynomial of the second block. Notice also that under the additional assumption of appropriate block-diagonality of the contemporaneous covariance matrix $\Sigma$, the $n_{2} \times 1$ subvector $x_{t}$ of $z_{t}=\left(y_{t}^{\prime}, x_{t}^{\prime}\right)^{\prime}$ is strongly exogenous. Multiplying the subsystem for $y_{t}$ by the adjoint matrix of $\Phi_{11}(L)$, one obtains the set of transfer functions (TF henceforth) for $y_{t}$, that is a set of dynamic equations with the same scalar AR polynomial, and vector moving averages in $x_{t}$ and in $\varepsilon_{1 t}$, with $\varepsilon_{i t}$ being the subvector of $\varepsilon_{t}$ corresponding to the partitioning of $z_{t}$ as $\left(y_{t}^{\prime}, x_{t}^{\prime}\right)^{\prime}$. These TF equations are conditional models which can be used to study the dynamics of $y_{t}$ or each of its components given $x_{t}$ and its past and the past of $y_{t}$.

Obviously, similar results can be obtained for the general case of block-diagonal or block-triangular systems with $k$ blocks.

\subsection{Interpretation in terms of a VMA index model}

This subsection further investigates the consequences of the presence of short-run co-movements for the VMA part of FEs. We consequently look at the adjoint matrix of $\Phi(L)$ and emphasize that the VMA representation follows a sort of multivariate index model (see Reinsel, 1983). In order to introduce the problem, let us look more closely at the adjoint matrix $\Phi(L)^{a d j}$, we have computed in Example 2 for $s=1$ SCCF relationship, i.e.

$$
\Phi(L)^{a d j} \varepsilon_{t}=\left[\begin{array}{cc}
1-0.2 L+0.6 L^{2} & -.2 L+.6 L^{2} \\
0.1 L-0.2 L^{2} & 1+0.1 L-0.2 L^{2}
\end{array}\right]\left[\begin{array}{l}
\varepsilon_{1 t} \\
\varepsilon_{2 t}
\end{array}\right] .
$$


We can also write the previous expression in terms of coefficient matrices and we observe the presence of a common right factor, namely of common right null spaces such that

$$
\Phi(L)^{a d j} \varepsilon_{t}=\left[\begin{array}{l}
\varepsilon_{1 t} \\
\varepsilon_{2 t}
\end{array}\right]+\left[\begin{array}{l}
-.2 \\
0.1
\end{array}\right]\left[\begin{array}{ll}
1 & 1
\end{array}\right]\left[\begin{array}{l}
\varepsilon_{1 t-1} \\
\varepsilon_{2 t-1}
\end{array}\right]+\left[\begin{array}{c}
0.6 \\
-0.2
\end{array}\right]\left[\begin{array}{ll}
1 & 1
\end{array}\right]\left[\begin{array}{l}
\varepsilon_{1 t-2} \\
\varepsilon_{2 t-2}
\end{array}\right]
$$

with the obvious numerical observation that $\Phi(L)^{a d j} \delta_{\perp}=\delta_{\perp}$, and $\delta_{\perp}^{\prime}=(1:-1)$. We now show that the VMA component of (3) has a structure that is analogous to the multivariate index model of Reinsel (1983), and this leads to the following general proposition:

Proposition 8 In a stationary $\operatorname{VAR}(p)$, the existence of $s$ SCCF vectors implies that in the FE representation the VMA coefficient matrices associated with degrees strictly larger than $(n-s-1) p$ have a common right null space that is spanned by $\delta_{\perp}$. Hence, post-multiplying the adjoint matrix $\Phi(L)^{\text {adj }}$ by $\delta_{\perp}$ reduces the order of the VMA component to a degree of at most $(n-s-1) p$ instead of $(n-s) p$.

Proof. We know from Proposition 3 that $\operatorname{det}[\Phi(L)]$ and $\Phi(L)^{\text {adj }}$ are both polynomials of order $(n-s) p$. Moreover, under SCCF we have that

$$
\Phi(L)=I_{n}-\sum_{j=1}^{p} \delta_{\perp} \Psi_{j}^{\prime} L^{j}
$$

where $\Phi_{j}=\delta_{\perp} \Psi_{j}^{\prime}$. Hence we can write

$$
\operatorname{det}[\Phi(L)] I_{n}=\Phi(L)^{a d j} \Phi(L)=\Phi(L)^{a d j}-\left[\Phi(L)^{a d j} \delta_{\perp}\right] \sum_{j=1}^{p} \Psi_{j}^{\prime} L^{j}
$$

from which it follows that $\Phi(L)^{a d j} \delta_{\perp}$ is a polynomial of order $(n-s-1) p$.

Corollary 9 In the particular case with $n-1=s$, the VMA component of the FEs follows an index model as in Reinsel (1983).

The last result is more a mathematical curiosity than a device to be used in an empirical analysis. We might think to use it for fully efficient estimation of the FEs for instance. But this shows that there exists a factor structure in the VMA component. In empirical investigations, this result can partially explain why the MA order is often found to be smaller than what it should be according to the theoretical implied models in Tables 1 and 2. Consider, for instance, the case with $w_{t}=\varepsilon_{t}+H_{1} \varepsilon_{t-1}$, where $w_{t}=\Phi(L)^{a d j} \varepsilon_{t}$. The first autocovariance of $w_{t}=\operatorname{det}[\Phi(L)] z_{t}$ is then given by $E\left(w_{t-1} w_{t}^{\prime}\right)=H_{1} \Sigma$ and, since $\delta_{\perp}$ spans the right null space of $H_{1}$, we have $E\left(w_{t-1} w_{t}^{\prime}\right)=0$ when $\Sigma=\delta_{\perp} \Lambda \delta_{\perp}^{\prime}$, where $\Lambda$ is a symmetric semi-positive definite matrix. Of course, under the condition $\Sigma=\delta_{\perp} \Lambda \delta_{\perp}^{\prime}$ the VAR innovations would be perfectly collinear and this cannot occur in practice. However, it might well be that $\Sigma \approx \delta_{\perp} \Lambda \delta_{\perp}^{\prime}$, and we label this case as a near coincidental situation. 
Figure 1: Quarterly growth rates of industrial production indexes (industrial sector)

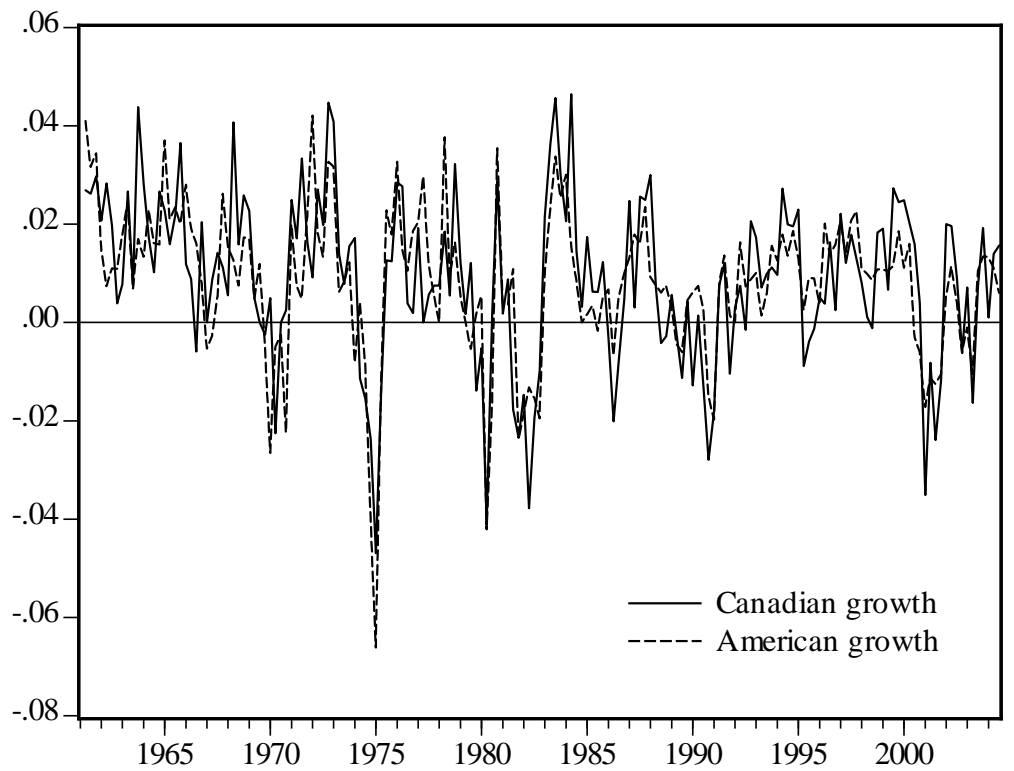

\subsection{An example: Industrial output growth in Canada and the US}

Engle and Kozicki (1993) find that there exists a SCCF relationship between the Canadian and the US quarterly growth rates of output (seasonally adjusted series). They have considered a sample from the late 1950s to the late 1990s. We select the same countries and we use the seasonally adjusted industrial production indexes from OECD main indicators over the period 1960:Q1-2004:Q3, namely we have 175 observations. Figure 1 plots these series. The model selection criteria LR, AIC, HQ and SBC lead to selecting $p=2$ for the log-levels of the bivariate processes. We reject the presence of cointegration at usual significance levels using Johansen's trace test. Consequently the analysis is performed in first differences, namely with quarterly growth rates, with one lag only.

The estimation by OLS of the VAR(1) in first differences delivers (standard errors in brackets)

$$
\left[\begin{array}{c}
\Delta \ln S A_{t} \\
\Delta \widehat{\ln C} A_{t}
\end{array}\right]=\left[\begin{array}{c}
0.003 \\
(0.001) \\
0.003 \\
(0.001)
\end{array}\right]+\left[\begin{array}{cc}
0.333 & 0.273 \\
(0.088) & (0.079) \\
0.265 & 0.360 \\
(0.102) & (0.092)
\end{array}\right]\left[\begin{array}{c}
\Delta \ln U S A_{t-1} \\
\Delta \ln C A_{t-1}
\end{array}\right]
$$

In order to check whether the coefficient matrix is of reduced rank we compute a SCCF test statistics using a canonical correlations approach (e.g. Vahid and Engle, 1993) between $\Delta z_{t}=\left(\Delta \ln U S A_{t}: \Delta \ln C A_{t}\right)^{\prime}$ and $\Delta z_{t-1}$. The results are as follows: $p-$ value $=0.31$ is the p-value associated with the null hypothesis $\left(\chi_{(1)}^{2}\right)$ that a linear combination of $\Delta z_{t}$ is orthogonal to the past of $\Delta z_{t}$. Information criteria also lead to 
select $s=1$ (Hecq, 2006). The estimated common cyclical feature relationship is $\Delta \ln U S A_{t}-1.05 \Delta \ln C A_{t}$. Without any constraints on the short-run, both implied series should follow $\operatorname{ARMA}(2,1)$ processes. However, the sample ACF and PACF indicate (figures not reported in the paper) that the orders are probably shorter and that $\mathrm{AR}(1)$ models for industrial production in both countries are more appropriate. For univariate $\mathrm{AR}(1)$ models, the estimated equations have quite similar AR roots

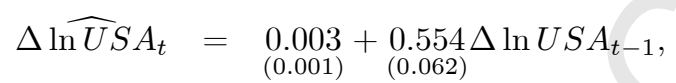

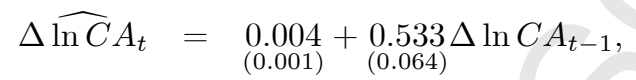

where for both equations, the null of no disturbance autocorrelation is not rejected using LM tests.

One part of our story is confirmed, i.e., the implied individual ARMA models have smaller AR orders than those that we should get in absence of common feature restrictions and the two series exhibit one significant SCCF relationship. Moreover, the AR roots are similar for both countries. What might look as evidence against our prediction is the absence of a MA component because both growth rates should be $\operatorname{ARMA}(1,1){ }^{6}$ However, since $\hat{\delta}$ is close to $(1:-1)^{\prime}$, the variances of VAR residuals are similar, and the correlation of VAR residuals is around 0.65, the results presented in Subsection 2.4 may explain why the MA(1) components are almost negligible.

\section{Estimation procedures for implied univariate models and mod- elling considerations}

As mentioned above, the finding that a set of series have identical autoregressive polynomials is an indication that these series have been generated by a VAR with non-(block)-diagonal and non-(block)-triangular matrix $\Phi(L)$ and that beyond having this common univariate autoregressive polynomial, they share other common features. ${ }^{7}$ This indication for the existence of common features calls for testing whether the moving average part of the set of variables $z_{i t}$ with identical autoregressive polynomials exhibits the multivariate index structure implied by the presence of common features (see Subsection 2.4). A test of the multivariate index structure could be carried out using the residuals of $\operatorname{det}[\Phi(L)] z_{i t}$. The properties of such a residual-based test would be affected by the properties of the estimator of the univariate autoregressive polynomials $\phi_{i}(L)$. It is therefore important to estimate these polynomials as accurately as possible.

We address the problem of estimation and testing of univariate ARMA models implied by a $\operatorname{VAR}(p)$ model with cofeature restrictions. Univariate ARMA models can be estimated in a straightforward way by the maximum likelihood method, that is we identify and estimate by ML for each series individually the

\footnotetext{
${ }^{6}$ Using univariate maximum likelihood estimation, the MA(1) component has p-values of 0.16 and 0.32 for respectively the US and Canada.

${ }^{7}$ Of course variables $z_{i t}$ with different univariate autoregressive polynomials could be generated by different sub-systems of a multivariate VAR with a corresponding block-diagonal matrix $\Phi(L)$.
} 
parsimonious empirical $\operatorname{ARMA}\left(p_{i}, q_{i}\right)$ such that

$$
z_{i t}=\hat{\alpha}_{i}+\Sigma_{j=1}^{p_{i}} \hat{\phi}_{i j} z_{i t-j}+\Sigma_{k=1}^{q_{i}} \hat{\theta}_{i k} \hat{u}_{i t-k}+u_{i t}, \quad i=1 \ldots n, t=1 \ldots T
$$

where $\hat{\alpha}_{i}, \hat{\phi}_{i j}$ and $\hat{\theta}_{i k}$ are estimated scalar parameters for series $i, i \in\{1,2, \ldots, n\} ; p_{i}$ and $q_{i}$ are the lag orders of the ARMA model for the $i$ th series and they might empirically differ from series to series. This first stage of the analysis is helpful because we can get a first idea about a maximum AR order $p$ as well as of the number of series that might share co-movements. ${ }^{8}$ In doing so, we obtain a rough indication, a sort of upper bound say, regarding the possible number of common feature vectors, with $\max \left(p_{i}\right)=(n-s) p$. Also, if for a series the AR order differs much from that for other series, then probably it does not share a common cycle with these other variables. Indeed, in absence of cancellation of common roots in the ARMA representations, the $\phi_{i j}$ 's should be the same for all $z_{i t}$ 's.

For sets of univariate ARMA models derived from a stationary multivariate ARMA model, Wallis (1977) has considered maximum likelihood estimation, whereas Palm and Zellner (1980) have considered both maximum likelihood and efficient two-step estimation. For (difference)-stationary series, the asymptotic properties of univariate and multivariate estimation and test procedures are standard and known. For integrated processes with or without cointegration present, when the unit root is not imposed, the asymptotic procedures generally are non-standard. Whether standard asymptotics hold or not, likelihood and efficient two-step methods for systems may be cumbersome to implement when the set of variables is large. Therefore, for empirical work, it will be useful to have tools at the disposal which can be easily implemented and lead to reliable inference. Satisfying this need is the objective of this section.

\subsection{Estimation of the common autoregressive polynomial using aggregates of individual series}

It has been emphasized in Section 2 that the series implied by the VAR must have the same AR coefficients. Consequently we investigate whether imposing this restriction using an estimator of the common AR part ${ }^{9}$ such that

$$
z_{i t}=\hat{\alpha}_{i}+\Sigma_{j=1}^{p_{i}} \hat{\phi}_{j} z_{i t-j}+\Sigma_{k=1}^{q_{i}} \hat{\theta}_{i k} \hat{u}_{i t-k}+\hat{u}_{i t}
$$

where $\hat{\phi}_{j}$ is the $j$ th lag order common coefficient to all $n$ that should be preferred to estimating ARMA models for the individual series. In order to avoid new notation, we use the same symbols as in (7) for the estimated intercept and moving average parameters.

The intuition underlying the use of (8) is twofold. First, under the null hypothesis, this is a correct way to impose the commonality observed in different series. Secondly, under the alternative namely when including a set of $n_{1}$ variables that does not belong to the same multivariate process then, erroneously imposing a

\footnotetext{
${ }^{8}$ Note that even though we do not identify the VAR order, we may nonetheless have an idea about $p$. For instance, with annual data $p$ is typically at most 4 but $p=2$ is the most common choice in empirical applications, whereas for quarterly data $p$ is rarely found to be larger than 9 .

${ }^{9}$ Note that the common AR operator can be obtained by factorizing the AR-polynomials for the individual series into the product of the individual operators and then cancelling common factors (Lütkepohl, 2005, 496).
} 
common AR root for the $n-n_{1}$ variables induces autocorrelation in the error terms. As a result, the detected order of the MA part can be higher than those observed for individual components. But before tackling this issue, we need to get estimates of the common AR component.

After having identified and estimated the parsimonious empirical $\operatorname{ARMA}\left(p_{i}, q_{i}\right)$ by $\mathrm{ML}$ for each series individually, one solution is to compute the average of $n$ AR parameters such that $\hat{\phi}_{j}^{m g}=n^{-1} \sum_{i=1}^{n} \hat{\phi}_{i j}$, $j=1 \ldots \max \left(p_{i}\right)$. However proceeding this way the homogeneity we have under the null is ignored. Moreover the estimation of $n$ equations by ML induces a lot of variability in the estimation of that average.

Instead, because the implied model for $\operatorname{det}[\Phi(L)] \bar{z}_{t}$ is at most a $\left.\mathrm{MA}[(n-s) p)\right]$ model in the presence of SCCF vectors for instance, we further use this observation and estimate an ARMA model for the average of the $n$ series in the ML estimation of

$$
\bar{z}_{t}=\hat{\alpha}-\Sigma_{j=1}^{p} \hat{\phi}_{j}^{a v} \bar{z}_{t-j}+\Sigma_{k=1}^{q} \hat{\theta}_{k} \hat{v}_{t-k}+\hat{v}_{t}
$$

with $\bar{z}_{t}=n^{-1} \sum_{i=1}^{n} z_{i t}$ the simple average of $n$ series, $v_{t}$ being the innovation of the univariate $\operatorname{ARMA}(p, q)$ for $\bar{z}_{t}$ and $\theta_{k}$ being the $k$-th lag parameter of the moving average part of $\bar{z}_{t}$. In this second setting we might use a LM test for no autocorrelation or graphical tools (i.e. ACF, PACF) on $\hat{v}_{t}$ to check the white noise hypothesis. A rejection of that null is a sign of misspecification, namely that we have likely included in the analysis a series that is not implied by the same system and consequently does not have the same final equation or does not have features in common with the other series in the system and therefore has higher degree AR and MA polynomials.

Cubadda et al. (2008) have shown by simulations that estimating a parsimonious ARMA model on aggregates is the preferred strategy for obtaining a common AR coefficient. Indeed, this procedure not only imposes the common AR parameters but also reduces the MA parts that might be annihilated by linearly combining the series (see Section 3). For instance, using the averages for Canada and the US with $\Delta \bar{z}_{t}=\frac{1}{2}\left(\Delta \ln U S A_{t}+\Delta \ln C A_{t}\right)$, the best model (using SBC) for the aggregates is the $\operatorname{AR}(1)$

$$
\Delta \bar{z}_{t}=\underset{(3.55)}{0.016}+\underset{(10.40)}{0.617} \Delta \bar{z}_{t-1}+\hat{e}_{t}
$$

where $t$-ratios are in brackets. LM autocorrelation tests on the residuals do not reject the null at any common significance level.

Moreover, aggregation can also yield an additional "virtuous" property for such models. Indeed, crosssectional aggregation implies a left multiplication of the adjoint by a vector, and this operation can reduce in some circumstances the order of the MA component of the aggregate. From equation (3), we see that the ARMA model for the aggregate is the following

$$
\operatorname{det}[\Phi(L)] \bar{z}_{t}=\kappa^{\prime} \Phi(L)^{a d j} \varepsilon_{t}
$$

where $\kappa=\frac{1}{n} \iota$, and $\iota$ is an $n$ vector of ones. Moreover, we know from Proposition 8 that the coefficient matrices of $\Phi(L)^{a d j}$ associated with degrees strictly larger than $(n-s-1) p$ have a reduced-rank structure 
with a common right null space that is spanned by $\delta_{\perp}$. Hence, it is possible that the polynomial matrix $\kappa^{\prime} \Phi(L)^{a d j}$ is, for instance, of order $(n-s) p-1$ instead of $(n-s) p$. This would require that the vector $\kappa$ lies in the left null space of the coefficient matrix of $\Phi(L)^{a d j}$ associated with degree $(n-s) p$, which is equal to $\Psi_{p \perp} G \delta^{\prime}$, where $G$ is a full rank $s \times s$ matrix, because of the relation

$$
\underbrace{\operatorname{det}[\Phi(L)]}_{(n-s) p} I_{n}=\Phi(L) \Phi(L)^{a d j}=\left(I_{n}-\sum_{j=1}^{p} \delta_{\perp} \Psi_{j}^{\prime} L^{j}\right) \underbrace{\Phi(L)^{a d j}}_{(n-s) p} .
$$

By similar reasoning, the order of the MA component of model (9) can be reduced till $(n-s-1) p$. This result explains why in empirical work it might occur that the MA order of the aggregate is even smaller than those of the individual series. Thus, we may interpret this occurrence as an evidence of the presence of co-movements, although the opposite conclusion is not valid.

\subsection{A Monte Carlo experiment on the estimator using aggregates}

Using the cross-sectional average is fine under the null that the FEs are from the same initial VAR model. In this subsection, we evaluate by simulation whether this is also true when we "erroneously substitute" to the group of variables having common cycles, additional series from another group. Next we look at alternative weighted cross-sectional averages of series having different standard deviations.

In both situations we look at the FEs of a VAR(1) with a reduced rank structure

$$
z_{t}=\alpha+\Phi_{1} z_{t-1}+\varepsilon_{t}=\alpha+\delta_{\perp} C_{1}^{\prime} z_{t-1}+\varepsilon_{t} .
$$

In order to keep fixed the AR and the MA orders in the $n$ implied models, we follow Cubadda et al. (2008) and impose that $s=n-1$ SCCF relationships, leading to $n$ implied $\operatorname{ARMA}(1,1)$ models for any $n$. The associated common feature matrix assumes full short-run convergence between economies (or variables). Indeed, one of the issues we want to address is to select a core of countries (or variables) with the maximum number of co-movements. The cofeature matrix has thus the following shape, for instance for $n=5$,

$$
\delta=\left(\begin{array}{rrrr}
1 & 0 & 0 & 0 \\
0 & 1 & 0 & 0 \\
0 & 0 & 1 & 0 \\
0 & 0 & 0 & 1 \\
-1 & -1 & -1 & -1
\end{array}\right)
$$

which leads to (up to a normalization) $\delta_{\perp}=(1: 1: 1: 1: 1)^{\prime} . \alpha$ is a $n$ dimensional vector of intercepts that we generate from a uniform distribution on $(0,1)$. In order to have the same AR coefficient whatever the odd number of individuals we choose $C_{1}^{\prime}=(0.5:-0.5: 0.5:-0.5: \cdots:-0.5,0.5)$. This guarantees both the stationarity of the multivariate process and the common value of the $\mathrm{AR}$ root $\left(\phi_{i 1}=0.5, i=1 \ldots n\right)$ for every series for any $n$. To show this we can simply use the property of partitioned matrices and compute 
$\operatorname{det}\left[I-\Phi_{1} L\right]$ when considering the $(n-1) \times(n-1)$ upper-left block of $\left(I-\Phi_{1} L\right)$. We use $M=2000$ replications of $T+50$ observations before dropping the first 50 points to initialize the random sequence.

\subsubsection{Experiment 1: Impact of adding series not generated from a VAR}

In this first experiment we successively consider $n=5,11,23$ series generated by the VAR (10) for $T=$ $50,100,250$. However, for the last $n_{2}=(n-1) / 2$ series, we substitute series generated by the following diagonal VAR:

$$
y_{t}=\Gamma_{1} y_{t}+\varepsilon_{t}, \quad t=1 \ldots T
$$

where $\Gamma_{1}=\operatorname{diag}(\gamma)+\mathrm{U}(-0.125,0.125)$, and $\gamma=0,0.25,0.5$. These are heterogeneous $\operatorname{AR}(1)$ processes such that only when $\gamma=0.5$ the coefficient comes from an uniform distribution centered around the same AR parameter of the FEs associated with (10). For the covariance matrix of the VAR errors we use in this first experiment $\Sigma_{\varepsilon}=I$. This implies cross-correlated errors $w_{t}=\Phi(L)^{a d j} \varepsilon_{t}$ in the FEs because their contemporaneous covariance matrix is $E\left(w_{t} w_{t}^{\prime}\right)=I+\Phi_{1} \Phi_{1}^{\prime}$. This corresponds to a correlation between the disturbances of the implied equations of $\rho=0.55$ for all pairs.

We estimate the common AR parameter using the parsimonious ARMA model on aggregates. Indeed, Cubadda et al. (2008) have found that this estimator has the best properties among various methods when the DGP is given by (10). Table 3 reports both the empirical bias and RMSE of this estimator when we generate $n_{1}=n_{2}-n_{1}$ series from system (10) and $n_{2}$ dimensional series from system (11). It emerges that the bias significantly increases if we include series with AR coefficients more distant from the final equation common parameters. However, the differences in terms of both bias and RMSE decrease as $n$ and $T$ increase.

TABLE 3

Estimation of a common autoregressive parameter $\phi_{i 1}=0.5$ in a mixed system

\begin{tabular}{lll|lll|lll}
\hline & & & \multicolumn{3}{|c|}{ Bias } & \multicolumn{3}{c}{ RMSE } \\
$n_{1}$ & $n_{2}$ & $\gamma$ & $T=50$ & $T=100$ & $T=250$ & $T=50$ & $T=100$ & $T=250$ \\
\hline \hline \multirow{2}{*}{2} & 2 & 0.50 & -0.074 & -0.034 & -0.010 & 0.196 & 0.124 & 0.068 \\
& & 0.25 & -0.100 & -0.060 & -0.036 & 0.214 & 0.139 & 0.078 \\
& & 0.00 & -0.122 & -0.078 & -0.056 & 0.225 & 0.152 & 0.093 \\
\hline \multirow{2}{*}{6} & 5 & 0.50 & -0.088 & -0.048 & -0.032 & 0.207 & 0.124 & 0.075 \\
& & 0.25 & -0.098 & -0.058 & -0.042 & 0.213 & 0.131 & 0.082 \\
& & 0.00 & -0.106 & -0.067 & -0.048 & 0.219 & 0.137 & 0.087 \\
\hline \multirow{2}{*}{12} & 11 & 0.50 & -0.080 & -0.041 & -0.015 & 0.207 & 0.137 & 0.067 \\
& & 0.25 & -0.083 & -0.043 & -0.018 & 0.212 & 0.136 & 0.068 \\
& & 0.00 & -0.085 & -0.044 & -0.020 & 0.213 & 0.137 & 0.069 \\
\hline
\end{tabular}

Note: A parsimonious empirical ARMA is chosen for the aggregates using SBC. The DGP

is such that $\mathrm{n}_{1}$ series are $\operatorname{ARMA}(1,1)$ with a common $\mathrm{AR}$ parameter equal to 0.5 and $\mathrm{n}_{2}$ series are $\mathrm{AR}(1)$ with a common coefficient equal to $\gamma+\mathrm{U}(-0.125,0.125)$. 


\subsubsection{Experiment 2: Simple vs. weighted averages}

In this second experiment, we extend the Monte Carlo analysis of Cubadda et al. (2008) to allow for heterogenous variances of the individual series. In particular, we now use in DGP (10) a covariance matrix of the VAR $\Sigma_{\varepsilon}$ such that its diagonal elements are $\left(1^{2}, 2^{2}, \ldots, n^{2}\right)$ and the correlation is equal to 0.7 for any pair of errors. This implies a contemporaneous covariance matrix $E\left(w_{t} w_{t}^{\prime}\right)=\Sigma_{\varepsilon}+\Phi_{1} \Sigma_{\varepsilon} \Phi_{1}^{\prime}$. We use $M=2000$ replications of $n=5,11,23$ individuals with $T=50,100,250$ observations after having discarded 50 points as starting values.

Since weighted averages are more appropriate than simple averages when series have different variances, we compare the performances of four types of aggregates:

1. $A v_{1}$ is a simple average of the series;

2. $A v_{2}$ is a weighted average where the weights are proportional to the inverse of the unconditional standard deviations of the series;

3. $A v_{3}$ is a weighted average where the weights are proportional to the inverse of the conditional standard deviations of the series $\sigma_{u_{i}}$ obtained through the estimated ARMA $(1,1)$ models;

4. $A v_{4}$ is a weighted average where the weights are proportional to the inverse of the standard deviations $\sqrt{\sigma_{u_{i}}^{2}\left(1+\theta_{i 1}^{2}\right)}$ of the MA(1) innovations of the series obtained through the estimated $\operatorname{ARMA}(1,1)$ models.

Table 4 reports the empirical bias and RMSE of the estimators of the common AR parameter for the above aggregates. It emerges that the estimator based on $A v_{4}$ has, overall, the best performance. The intuition behind this finding is that, since the MA component is noise in this estimation problem, the $A v_{4}$ weighted average gives more weight to less noisy series. However, the differences in terms of performance of the various estimators are not large, and they tend to disappear as both $n$ and $T$ become large. 
TABle 4

Various estimators of a common autoregressive parameter

\begin{tabular}{cc|ccc|ccc}
\hline & & \multicolumn{3}{|c|}{ Bias } & \multicolumn{3}{c}{ RMSE } \\
\hline \hline 5 & & $T=50$ & $T=100$ & $T=250$ & $T=50$ & $T=100$ & $T=250$ \\
\hline & $A v_{1}$ & -0.072 & -0.028 & -0.007 & 0.205 & 0.128 & 0.069 \\
& $A v_{2}$ & -0.054 & -0.012 & 0.011 & 0.199 & 0.127 & 0.069 \\
& $A v_{3}$ & -0.046 & -0.006 & 0.018 & 0.190 & 0.128 & 0.073 \\
& $A v_{4}$ & -0.040 & -0.001 & 0.019 & 0.183 & 0.122 & 0.073 \\
\hline \multirow{2}{*}{11} & $A v_{1}$ & -0.065 & -0.033 & -0.011 & 0.185 & 0.120 & 0.068 \\
& $A v_{2}$ & -0.054 & -0.022 & -0.001 & 0.182 & 0.115 & 0.067 \\
& $A v_{3}$ & -0.051 & -0.017 & 0.003 & 0.186 & 0.115 & 0.067 \\
& $A v_{4}$ & -0.046 & -0.015 & 0.005 & 0.177 & 0.113 & 0.067 \\
\hline 23 & $A v_{1}$ & -0.079 & -0.033 & -0.010 & 0.211 & 0.124 & 0.067 \\
& $A v_{2}$ & -0.077 & -0.029 & -0.006 & 0.216 & 0.123 & 0.066 \\
& $A v_{3}$ & -0.073 & -0.027 & -0.003 & 0.214 & 0.122 & 0.066 \\
& $A v_{4}$ & -0.067 & -0.025 & -0.003 & 0.201 & 0.121 & 0.065 \\
\hline
\end{tabular}

Note: A parsimonious empirical ARMA model is chosen for the aggregates using SBC.

\section{Analysis of per capita real GDP in Latin America}

We study the first differences of the log levels of the per capita real gross domestic product (namely the growth rates) of nine Latin American economies. We have used annual data from 1950 to 2002, i.e. 53 observations. The series are derived from the Total Economy Database. ${ }^{10}$ We have used on purpose the same series as in Hecq et al. (2006) because it emerged there that it was not obvious to determine a priori the variables to be included in a common trend, common cycle analysis. Table 5 reports the list of these countries as well as the univariate ARMA models and outliers we have identified using the software program Tramo/Seats implemented in Eviews 6 (see Gómez and Maravall, 1996). This illustrates the use of a well known alternative to ACF and PACF analyses with the advantage to determine the presence of aberrant values. We also report the estimates of the significant coefficients at a $10 \%$ level.

As far as short-run co-movements are concerned, we can first form a group of countries that includes Brazil, Columbia and Guatemala for which an $\operatorname{AR}(1)$ was found. ${ }^{11}$ With these three countries, $p=2$ for the $\log$ levels was sufficient to capture the dynamics using a VAR. Moreover we do not conclude to the existence of long-run cointegration relationships between the log of per capita real GDP using Johansen's trace tests. Consequently, the $\operatorname{VAR}(1)$ for first differences implies $\operatorname{ARMA}(3,2)$ models for growth rates. This is not compatible with the parsimonious $\operatorname{AR}(1)$ we identify from the data. We interpret this as a sign of the

\footnotetext{
${ }^{10}$ University of Groningen and The Conference Board, GGDC Total Economy Database www.eco.rug.nl/ggdc. The variables are expressed in 1990 US dollars and converted at "Geary-Khamis" purchasing power parities.

${ }^{11}$ Note that when looking for a set of countries sharing common cycles, white noise processes do not affect the order of the univariate ARMA processes of the remaining variables. This is because having one more white noise series included leads to consider the degree $(n+1-(s+1)) p=(n-s) p$ for the implied AR and MA parts for instance. But this simplifies the search procedure.
} 
presence of short-run co-movements. Indeed, $\operatorname{ARMA}(1,1)$ and $\operatorname{AR}(1)$ are compatible with $s=2$ short-run cofeature relationships. If we identify an ARMA model for the aggregate of the three countries using Tramo, the most parsimonious model is the $\operatorname{AR}(1)$ without outliers

$$
\Delta \bar{z}_{t}^{*}=\underset{(3.00)}{0.64}+\underset{(5.21)}{0.597} \Delta \bar{z}_{t-1}^{*}+\hat{e}_{t}
$$

where $\Delta \bar{z}_{t}^{*}$ is the weighted average $A v_{3}$ with estimated weights, which is obviously equal to $A v_{4}$ in this case, for which the residuals $\hat{e}_{t}$ show no sign of autocorrelation.

TABLE 5

\begin{tabular}{llll}
\multicolumn{4}{c}{ Identified $\operatorname{ARMA}(p, q)$ models } \\
\hline Identified components: & $\mathrm{AR}$ & $\mathrm{MA}$ & Outliers \\
\hline \hline Brazil & $1(0.51)$ & 0 & 1981 \\
Chile & 0 & $1(0.33)$ & 1982,1975 \\
Columbia & $1(0.39)$ & 0 & 1999 \\
Mexico & 0 & $1(0.44)$ & 1995 \\
Peru & 0 & $1(0.58)$ & 1983 \\
Venezuela & 0 & 0 & - \\
Argentina & 0 & $1(0.23)$ & - \\
Ecuador & 0 & 0 & 1987,1999 \\
Guatemala & $1(0.57)$ & 0 & - \\
\hline
\end{tabular}

In Table 5, we report the orders of the selected processes, the point estimates for the coefficients (between parentheses) and the years for which outliers have been detected and accounted for.

From these results we conjecture that there exist strong short-run co-movements characterized by a unique common cycle for that group of three countries. Both the individual ARMA orders and the absence of any autocorrelation in the residuals of the aggregated estimated equation support this conclusion. To have an alternative look at this issue (it is possible in this case because $n$ is small), we use SCCF test statistics for the number of cofeatures using a canonical correlation approach as well as the value of information criteria. We estimate the VAR after correcting for the outliers detected at the univariate level. The $p-v a l u e$ associated with $s=2$ is 0.2 and information criteria also select $s=2$ (see Hecq et al., 2006 and Hecq 2006).

\section{Conclusion}

This paper has derived the implications for the univariate processes of the presence of common factors or trends resulting from common cyclical features or cointegration in multivariate time series. Exploiting the information about common dynamics contained in individual series and exhibited in individual ARIMA estimations, a strategy has been proposed to study the presence of co-movements in large sets of series without the need to develop and jointly estimate a large complex multivariate model for these series. This 
strategy is based on the theoretical results for the orders of the FEs for VAR models with co-features. The strategy is shown to yield sensible results in an application involving GDP data for nine countries.

Many further developments are currently under investigations such as, inter alia, the development of panel unit root tests and the extension to seasonal models. Moreover, the small sample and asymptotic properties of the proposed methods must be more deeply evaluated.

The tools we introduce can be extended in several directions. For instance, they allow to study comovements between variables or convergence among economies, forecast series, and build business cycle indices without requiring a full parametric system with many variables. The advantages of our approach are: 1) its feasibility when it is not possible to jointly analyze a complete system or when we prefer to work using a sub-system, 2) its usefulness to detect sets of variables that are likely to be generated by seemingly unrelated subsystems for subsets of variables that have some features in common, 3) the accuracy of forecasts, 4) the ease of its implementation when a large number of jointly dependent variables has to be studied in complex situations, 5) the potential empirical applications in many fields.

Finally, the insights obtained from the analyses of single series and subset of series with identical AR parts, and from testing for short-run and long-run co-feature restrictions can be incorporated in the multivariate model for all series to be modeled.

\section{References}

[1] Aнn, S.K. (1997), Inference of Vector Autoregressive Models with Cointegration and Scalar Components, Journal of the American Statistical Association, 92, 350-356.

[2] Bai, J. And S. NG (2004), A PANIC Attack on Unit Roots and Cointegration, Econometrica 72, $1127-1177$.

[3] Brüggemann, R., Krolzig, H.M. and H. LÜtkepohl (2003), Comparison of Model Reduction Methods for VAR Processes, Economics Papers \#2003-W13, Economics Group, Nuffield College, University of Oxford.

[4] Centoni M., Cubadda G., And A. Hecq (2007), Common Shocks, Common Dynamics, and the International Business Cycle, Economic Modelling, 24, 149-166.

[5] Cubadda, G. and A. Hecq (2001), On Non-Contemporaneous Short-Run Comovements, Economics Letters, 73, 389-397.

[6] Cubadda, G. (2007), A Unifying Framework for Analyzing Common Cyclical Features in Cointegrated Time Series, Computational Statistics and Data Analysis, 52, 896-906

[7] Cubadda, G., Hecq, A. and F.C. Palm (2008), Macro-panels and Reality, Economics Letters, 99, $537-540$.

[8] Engle, R.F. and S. Kozicki (1993), Testing for Common Features (with comments), Journal of Business and Economic Statistics 11, 369-395. 
[9] Ericsson, N., 1993, Comment (on Engle and Kozicki, 1993), Journal of Business and Economic Statistics, 11, 380-383.

[10] Forni M., M. Hallin, Lippi M. And L. Reichlin (2000), The Generalized Dynamic Factor Model: Identification and Estimation, Review of Economics and Statistics, 82, 4, 540-554.

[11] Franses, P.H. (1998), Time Series Models for Business and Economic Forecasting, Cambridge University Press.

[12] Gómez, V. and A. Maravall (1996), Programs TRAmo (Time series Regression with Arima noise, Missing observations, and Outliers) and SEATS (Signal Extraction in Arima Time Series). Instructions for the User, Working Paper 9628, Research Department, Banco de España.

[13] Gouriéroux, Ch. and I. Peaucelle (1989), Detecting a Long-run Relationship, CEPREMAP Discussion Paper, 8902.

[14] Hecq, A. (2006), Cointegration and Common Cyclical Features in VAR Models: Comparing Small Sample Performances of the 2-Step and Iterative Approaches, University of Maastricht Research Memorandum.

[15] Hecq, A., Palm, F.C. and J.P. Urbain (2002), Separation, weak exogeneity and P-T decomposition in cointegrated VAR systems with common features, Econometric Reviews, 21, 273-307.

[16] Hecq, A., Palm, F.C. and J.P. Urbain (2006), Common Cyclical Features Analysis in VAR Models with Cointegration, Journal of Econometrics, 132, 117-141.

[17] Johansen, S. (1996), Likelihood-Based Inference in Cointegrated Vector Autoregressive Models (Oxford University Press: Oxford).

[18] LÜtкepohl, H. (2005), New Introduction to Multiple Time Series Analysis, Springer Verlag, Berlin.

[19] Maravall, A. and A. Mathis (1994), Encompassing Univariate Models in Multrivariate Time Series: a Case Study, Journal of Econometrics, 61, 197-233.

[20] Palm, F.C. (1977), On Univariate Time Series Methods and Simultaneous Equation Econometric Models, Journal of Econometrics, 5, 379-388.

[21] Palm, F.C. and A. Zellner (1980), Large-Sample Estimation and Testing Procedures for Dynamic Equation Systems, Journal of Econometrics, 12, 251-283.

[22] Quenoullle, M.H. (1957, 1968 2ND ED), The Analysis of Multiple Time-Series, Hafner Publishing Co., New-York.

[23] Reinsel, G. (1983), Some Results on Multivariate Autioregressive Index Models, Biometrika, 70, $145-156$. 
[24] Schleicher, Сн. (2007), Codependence in Cointegrated Autoregressive Models, Journal of Applied Econometrics, 22, 137-159.

[25] Stock J.H. And M.W. Watson (2002), Macroeconomic Forecasting Using Diffusion Indexes, Journal of Business and Economic Statistics, 20, 2, 147-162.

[26] Tiao, G.C. and R.S. Tsay (1989), Model Specification in Multivariate Time Series (with comments), Journal of Royal Statistical Society, Series B, 51, 157-213.

[27] Vahid, F. and R.F. Engle (1993), Common Trends and Common Cycles, Journal of Applied Econometrics, 8, 341-360.

[28] Vahid, F. and R.F. Engle (1997), Codependent Cycles, Journal of Econometrics, 80, 199-221.

[29] Wallis, K.F. (1977), Multiple Time Series Analysis and the Final Form of Econometric Models, Econometrica, 45, 1481-97.

[30] Zellner, A. and F.C. Palm (1974), Time Series Analysis and Simultaneaous Equation Econometric Models, Journal of Econometrics, 2, 17-54.

[31] Zellner, A. And F.C. Palm (1975), Time Series and Structural analysis of Monetary Models of the US Economy, Sanhya: The Indian Journal of Statistics, Series C, 37, 12-56.

[32] Zellner, A. and F.C. Palm (2004), The Structural Econometric Time Series Analysis Approach, Cambridge University Press. 